\title{
A hybrid method combining the FDTD and a time domain boundary-integral equation marching-on-in-time algorithm
}

\author{
A. Becker and V. Hansen
}

Lehrstuhl für Theoretische Elektrotechnik der Universität Wuppertal, Rainer-Gruenter-Str. 21, 42119 Wuppertal, Germany

\begin{abstract}
In this paper a hybrid method combining the FDTD/FIT with a Time Domain Boundary-Integral Marching-on-in-Time Algorithm (TD-BIM) is presented. Inhomogeneous regions are modelled with the FIT-method, an alternative formulation of the FDTD. Homogeneous regions (which is in the presented numerical example the open space) are modelled using a TD-BIM with equivalent electric and magnetic currents flowing on the boundary between the inhomogeneous and the homogeneous regions. The regions are coupled by the tangential magnetic fields just outside the inhomogeneous regions. These fields are calculated by making use of a Mixed Potential Integral Formulation for the magnetic field. The latter consists of equivalent electric and magnetic currents on the boundary plane between the homogeneous and the inhomogeneous region. The magnetic currents result directly from the electric fields of the Yee lattice. Electric currents in the same plane are calculated by making use of the TD-BIM and using the electric field of the Yee lattice as boundary condition. The presented hybrid method only needs the interpolations inherent in FIT and no additional interpolation. A numerical result is compared to a calculation that models both regions with FDTD.
\end{abstract}

\section{Introduction}

The FDTD-Method is a very efficient and accurate technique for solving bounded electromagnetic field problems. For the analysis e.g. of scattering or antenna problems including the open space the solution space must be kept finite by introducing an absorbing boundary condition (ABC). An often applied $A B C$ is obtained by introducing a perfectly matched layer (PML), the effect of which is incorporated into the solution-procedure by regarding the PML-region as a part of the FDTD solution space, that means, by treating it within the FDTD scheme. As the FDTD is a "local" method the PML

Correspondence to: A. Becker

(abecker@uni-wuppertal.de) can be regarded as a "local" $\mathrm{ABC}$, too. A "global" $\mathrm{ABC}$ is obtained if an integral formulation for a surface inclosing the bounded FDTD solution space is used as a starting point. As an example for such a "global" FDTD-ABC formulation (de Moerlosse et al., 1993) shall be mentioned, where the magnetic field is calculated just outside the FDTD solution space by an integral equation. As the Yee-scheme consisting of two shifted lattices does not provide the required electric and magnetic currents in the same plane, one of these must be calculated by an additional interpolation. A very interesting class of TD-integral formulations are the so called Time Domain Boundary-Integral Marching-on-in-Time Algorithms (TD-BIMs), which in the last few years draw more an more attention. By this, the two main drawbacks of TDBIMs -stability problems and high computational cost- have been more and more alleviated (e.g. Shanker et al., 2000). Thus, it is reasonable to make use of these advantages and to develop a hybrid method that combines the FDTD/FIT and a TD-BIM, in other words, to make use of the TD-BIM as a "global" ABC for the FDTD solution space.

This paper is organized as follows:

- Section 2 gives an overview of the TD-BIM that is used here to calculate the electric current density at the boundary between the inhomogeneous and the homogeneous body.

- Section 3 shortly repeats the differences between the FDTD and the FIT. The latter is used here to model inhomogeneous bodies.

- Section 4 explains the algorithm of the hybrid method proposed here which combines both numerical techniques.

- Section 5 shows a first numerical example to test the method.

- In Sect. 6 a summary and an outlook are given. 

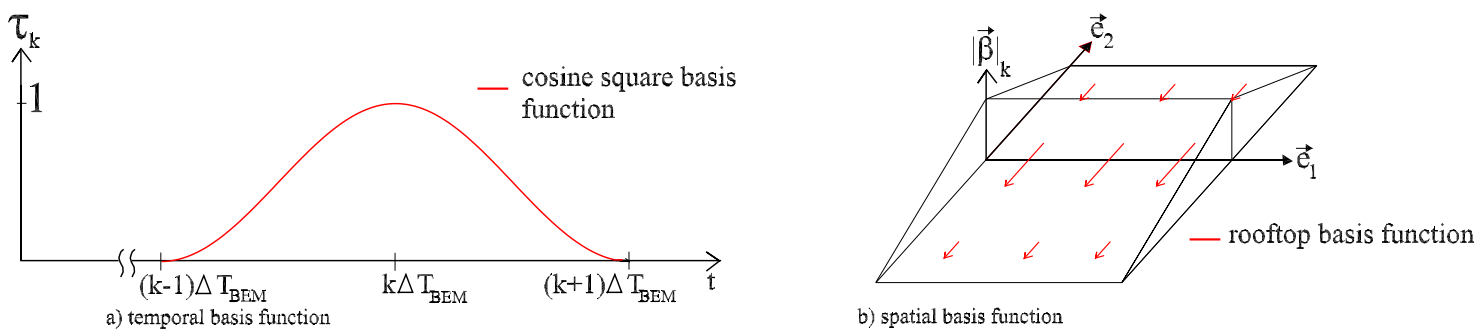

b) spatial basis function

Fig. 1. Temporal and spatial basis function used for the TD-BIM.
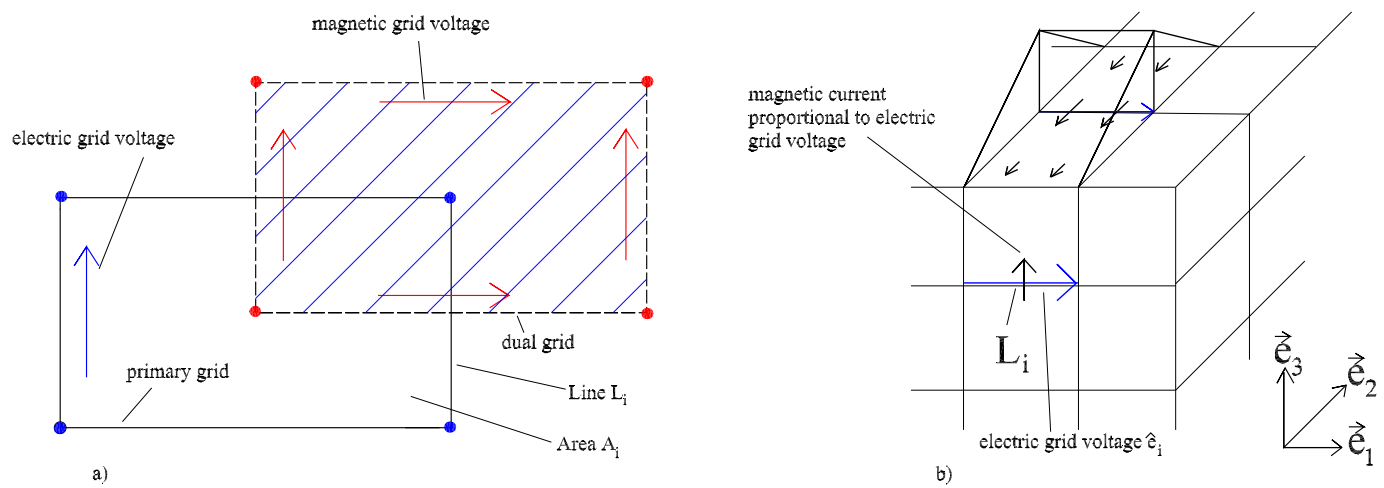

Fig. 2. Spatial allocation of the electric and the magnetic grid voltages and the straightforward allocation of the magnetic current.

\section{TD-BIM}

The time domain electric field integral equation (TD-EFIE) for a homogeneous infinite space is given to:

$\boldsymbol{E}(\boldsymbol{r}, t)=-\frac{\partial \boldsymbol{A}(\boldsymbol{r}, t)}{\partial t}-\nabla \phi(\boldsymbol{r}, t)-\frac{1}{\varepsilon} \nabla \times \boldsymbol{F}(\boldsymbol{r}, t)$.

When applying the equivalence principle the potentials are related to the equivalent surface sources of the field by

$\boldsymbol{A}(\boldsymbol{r}, t)=\frac{\mu}{4 \pi} \int_{A^{\prime}} \frac{\boldsymbol{J}\left(\boldsymbol{r}^{\prime}, t-\frac{R}{c}\right)}{R} d a^{\prime}$,

$\phi(\boldsymbol{r}, t)=\frac{1}{4 \pi \varepsilon} \int_{A^{\prime}} \frac{\rho\left(\boldsymbol{r}^{\prime}, t-\frac{R}{c}\right)}{R} d a^{\prime}$,

$\boldsymbol{F}(\boldsymbol{r}, t)=\frac{\varepsilon}{4 \pi} \int_{A^{\prime}} \frac{\boldsymbol{M}\left(\boldsymbol{r}^{\prime}, t-\frac{R}{c}\right)}{R} d a^{\prime}$

with $R=\left|\boldsymbol{r}-\boldsymbol{r}^{\prime}\right|$ and the region outside of $A^{\prime}$ source free. The surface $A^{\prime}$ is the surface on which the currents flow, the so called Huygens-surface. The substitution of Eq. (2), Eq. (3) (together with the continuity equation $\nabla \boldsymbol{J}+\frac{\partial}{\partial t} \rho=0$ ) and Eq. (4) into Eq. (1) results into a relationship between the electric field and the electric and the magnetic current densities (Rao, 1999):

$$
\begin{aligned}
& \boldsymbol{E}(\boldsymbol{r}, t)=\int_{A^{\prime}}\left[-\frac{\mu}{4 \pi} \frac{\partial}{\partial t} \frac{\boldsymbol{J}\left(\boldsymbol{r}^{\prime}, t-\frac{R}{c}\right)}{R}\right. \\
& \left.+\nabla \frac{\int_{0}^{t-\frac{R}{c}} \nabla \boldsymbol{J}\left(\boldsymbol{r}^{\prime}, t\right) d t}{4 \pi \varepsilon R}-\nabla \times \frac{\boldsymbol{M}\left(\boldsymbol{r}^{\prime}, t-\frac{R}{c}\right)}{4 \pi R}\right] d a^{\prime} .
\end{aligned}
$$

For the numerical solution the surface $A^{\prime}$ is subdivided into small patches. The electric and magnetic current density is numerically approximated as a series of unknown coefficients multiplied with basis functions:

$$
\begin{aligned}
& \boldsymbol{J}(\boldsymbol{r}, t)=\sum_{i=0}^{N_{s}} \sum_{j=0}^{N_{t}} J_{(i, j)} \boldsymbol{\beta}_{i}(\boldsymbol{r}) \tau_{j}(t), \\
& \boldsymbol{M}(\boldsymbol{r}, t)=\sum_{i=0}^{N_{s}} \sum_{j=0}^{N_{t}} M_{(i, j)} \boldsymbol{\beta}_{i}(\boldsymbol{r}) \tau_{j}(t)
\end{aligned}
$$

$N_{s}$ and $N_{t}$ are the numbers of space samples and the time step in which the electric field is calculated, respectively. The spatial and temporal basis functions applied here are shown in Fig. 1. The width of the temporal basis function is $2 \triangle T_{B E M}$. We integrate the combination of Eqs. (5) and (6) multiplied with a test function $\boldsymbol{\beta}_{k}$ over the domain $A_{k}$ of the test function (Sarkar et al., 2000):

$$
\begin{aligned}
& \int_{A_{k}} \boldsymbol{E}(\boldsymbol{r}, t) \boldsymbol{\beta}_{k}(\boldsymbol{r}) d a=\int_{A_{k}} \int_{A^{\prime}} \sum_{i=0}^{N_{s}} \sum_{j=0}^{N_{t}} \\
& {\left[-\frac{\mu}{4 \pi} \frac{J_{(i, j)} \boldsymbol{\beta}_{k}(\boldsymbol{r}) \boldsymbol{\beta}_{i}\left(\boldsymbol{r}^{\prime}\right) \tau^{\prime}\left(t-\frac{R}{c}\right)}{R}\right.} \\
& -\frac{\int_{0}^{t-\frac{R}{c}} J_{(i, j)} \gamma_{k}(\boldsymbol{r}) \gamma_{i}\left(\boldsymbol{r}^{\prime}\right) \tau(t) d t}{4 \pi \varepsilon R} \\
& \left.-\nabla \times \frac{M_{(i, j)} \boldsymbol{\beta}_{k}(\boldsymbol{r}) \boldsymbol{\beta}_{i}\left(\boldsymbol{r}^{\prime}\right) \tau\left(t-\frac{R}{c}\right)}{4 \pi R}\right] d a^{\prime} d a .
\end{aligned}
$$




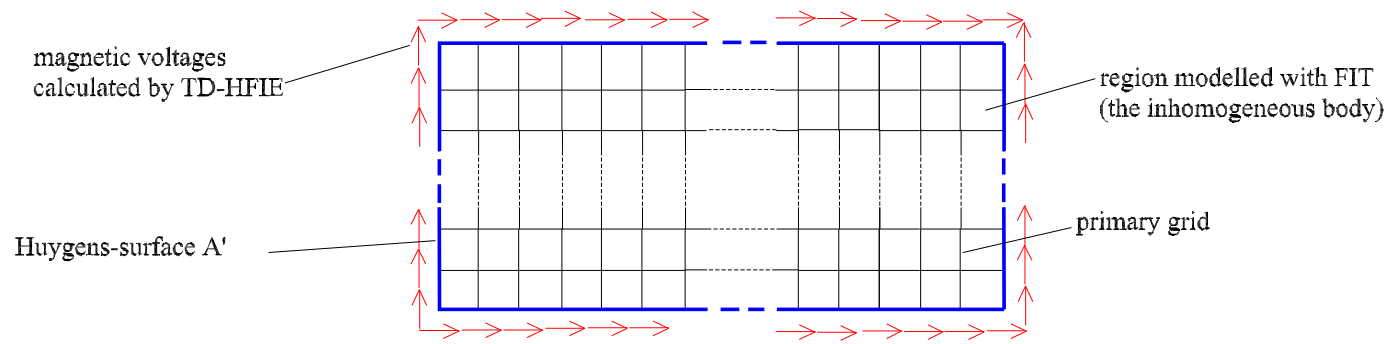

Fig. 3. The allocation of the Huygens-surface $A^{\prime}$ in the Yee lattice.

The term $\gamma$ represents the surface-divergence of the basis function $\boldsymbol{\beta}$. Equation (7) connects the tangential electric field on surface $A_{k}$ to the electric and the magnetic currents flowing on surface $A^{\prime}$. The equivalence principle relates the tangential electric field on the surface $A_{k}$ to the equivalent magnetic current:

$\boldsymbol{\beta}_{k} \cdot(\boldsymbol{n} \times \boldsymbol{M})=\boldsymbol{\beta}_{k} \cdot(\boldsymbol{n} \times(\boldsymbol{E} \times \boldsymbol{n}))$

$=\boldsymbol{\beta}_{k} \cdot[\boldsymbol{E}(\boldsymbol{n} \cdot \boldsymbol{n})-\boldsymbol{n} \cdot(\boldsymbol{n} \cdot \boldsymbol{E})]=\boldsymbol{\beta}_{k} \cdot \boldsymbol{E}$

Substituting Eq. (8) into Eq. (7) leads to a relationship between the electric and magnetic current density:

$$
\begin{aligned}
& \int_{A_{k}} \sum_{i=1}^{N} M_{\left(i, N_{t}\right)} \boldsymbol{\beta}_{k} \cdot\left(\boldsymbol{n} \times \boldsymbol{\beta}_{i}(\boldsymbol{r})\right) d a= \\
& \int_{A_{k}} \int_{A^{\prime}} \sum_{i=1}^{N} \sum_{j=1}^{N_{t}}\left[-\frac{\mu}{4 \pi} \frac{J_{(i, j)} \boldsymbol{\beta}_{k}(\boldsymbol{r}) \boldsymbol{\beta}_{i}\left(\boldsymbol{r}^{\prime}\right) \tau^{\prime}\left(t-\frac{R}{c}\right)}{R}\right. \\
& \left.-\frac{\int_{0}^{t-\frac{R}{c}} J_{(i, j)} \gamma_{k}(\boldsymbol{r}) \gamma_{i}\left(\boldsymbol{r}^{\prime}\right) \tau(t) d t}{4 \pi \varepsilon R}\right] d a^{\prime} d a .
\end{aligned}
$$

If the actual magnetic current density on surface $A^{\prime}$ is known, the actual electric current density can be calculated from the electric current density retarded in time at least one time step and from the magnetic current density by enforcing continuity on all subdomains $A_{k}$ of $A^{\prime}$. Eq. (9) can be written as:

$$
\begin{aligned}
& \underbrace{\left(\begin{array}{ccc}
Z_{1,1} & \ldots & Z_{1, N} \\
\vdots & \ddots & \vdots \\
Z_{N, 1} & \ldots & Z_{N, N}
\end{array}\right)}_{Z}\left(\begin{array}{c}
I_{1, N_{t}} \\
\vdots \\
I_{N, N_{t}}
\end{array}\right) \\
& =\boldsymbol{f}\left(\sum_{i=1}^{N} \sum_{j=1}^{N_{t}-1} J_{(i, j)}\right)+\boldsymbol{g}\left(\sum_{i=1}^{N} \sum_{j=1}^{N_{t}} M_{(i, j)}\right) \\
& =\left(\begin{array}{c}
\sum_{i=1}^{N} \sum_{j=1}^{N_{t}-1} Y_{(1, i, j)} J_{(i, j)}+\sum_{i=1}^{N} \sum_{j=1}^{N_{t}} X_{(1, i, j)} M_{(i, j)} \\
\vdots \\
\sum_{i=1}^{N} \sum_{j=1}^{N_{t}-1} Y_{(N, i, j)} J_{(i, j)}+\sum_{i=1}^{N} \sum_{j=1}^{N_{t}} X_{(N, i, j)} M_{(i, j)}
\end{array}\right)
\end{aligned}
$$

The matrix $\mathbf{Z}$ describes the actual electric fields produced by the actual electric currents and thus is highly sparse. In a time-invariant system both $Z$ and the coefficients $X_{(k, i, j)}$ and $Y_{(k, i, j)}$ have to be calculated just once, at the first time step. If the tangential electric field equals zero (that means the material is a perfect electric conductor and there is no magnetic current density) Eq. (10) will be sufficient for calculating the electric current density in a marching-on-in-time algorithm (MoT). Otherwise a second equation is needed to calculate both current densities. In our case we will use the FIT to update the magnetic current density and Eq. (10) to update the electric current density. Thus the surface $A^{\prime}$ will encase the region modelled with FIT.

\section{FDTD/FIT}

The TD-FIT can be considered as a special formulation of the FDTD-method (Weiland, 1996). In contrast to FDTD it is based on the Maxwell Equations in integral form. By using electric and magnetic fluxes and grid voltages as unknown coefficients and by using the Yee lattice to allocate them, these equations can be written as:

$$
\begin{aligned}
& \boldsymbol{C} \hat{\boldsymbol{e}}=-\frac{\partial}{\partial t} \hat{\hat{\boldsymbol{b}}} \\
& \tilde{\boldsymbol{C}} \hat{\boldsymbol{h}}=\frac{\partial}{\partial t} \hat{\hat{\boldsymbol{d}}}+\hat{\hat{j}}
\end{aligned}
$$

$\hat{\boldsymbol{e}}$ represents the grid voltage above one Line $L_{i}$ of the Yee cell. The term $\hat{\hat{d}}$ represents the flux through one Area $A_{i}$ of the Yee cell. The unknown coefficients can be considered as the analytic - and thus numerically error-free - values. The application of Eq. (12) is illustrated in Fig. 2a. A second order accurate central difference approximation for the time derivative leads to the leap frog algorithm. The definition of grid voltages as unknown coefficients is essential if using the FIT instead of FDTD for our hybrid method.

\section{Hybrid method}

In this section we will introduce our hybrid method. The TDBIM acts as a "global" absorbing boundary condition enclosing the inhomogeneous bodies which are modelled by FIT. 


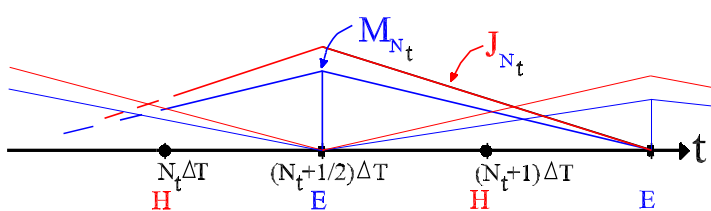

a)

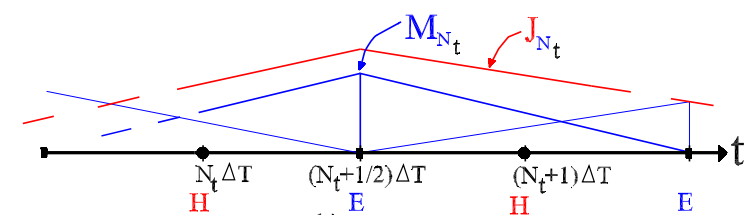

b)

Fig. 4. The temporal allocation of the magnetic currents and possible allocations of the electric currents.

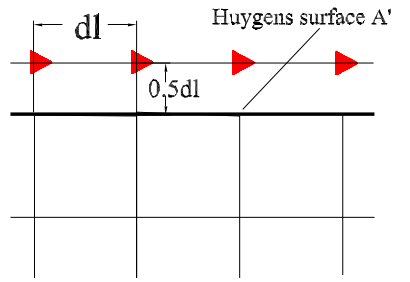

a)

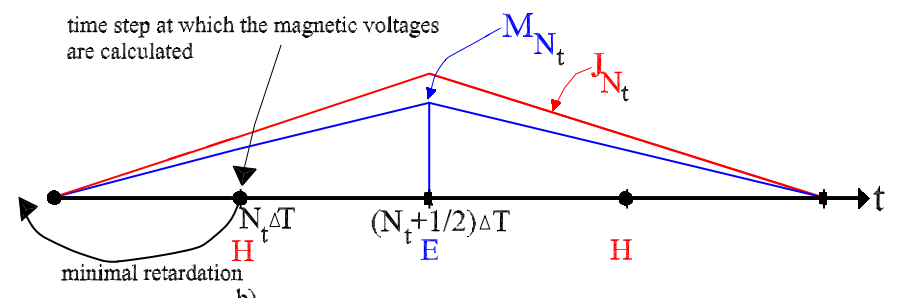

b)

Fig. 5. The boundary of the FIT-domain and the resulting minimal retardation in the calculation of the magnetic grid voltages.

\subsection{Basic features}

We interconnect the inhomogeneous body and the surrounding homogeneous region by calculating tangential magnetic grid voltages just outside the inhomogeneous body using a TD magnetic field integral equation (TD-HFIE) which uses electric and magnetic currents flowing on the Huygenssurface $A^{\prime}$ whose location is shown in Fig. 3 .

The magnetic current on the surface $A^{\prime}$ results directly from the electric grid voltages of the FIT, which will be shown beneath. Due to the fact that the magnetic grid voltages are spatially (and temporally) separated from the electric grid voltages the electric currents on the Huygens-surface $A^{\prime}$ can not be calculated from the magnetic grid voltages. Therefore the electric current will be calculated by using a TD-BIM -as described in Sect. 2 - which is capable of considering bodies outside the FIT volume.

To develop the algorithm of the hybrid method we start with the FIT and a Cartesian grid. The unknowns in FIT are grid voltages defined as:

$\hat{e}_{i}=\int_{L_{i}} \boldsymbol{E} d \boldsymbol{s}, \hat{h}_{i}=\int_{L_{i}} \boldsymbol{H} d \boldsymbol{s}$

As already mentioned we use the rooftop basis function for the TD-BIM because the discretization for FIT leads to a patch model of the surface $A^{\prime}$ consisting of rectangular elements (see Fig. 2) if Cartesian Coordinates are used. The unknowns in the TD-BIM are proportional to the flux orthogonal to these edges. With the location in time of the magnetic current density according to Fig. 4 this results into the following relationship between the unknown $\hat{e}_{i}$ of the FIT and the magnetic current of the TD-BIM (see Fig. 2b):

$$
\begin{aligned}
& \int_{L_{i}} \boldsymbol{M}_{N_{t}} \boldsymbol{e}_{3}=\int_{L_{i}}\left(\boldsymbol{e}_{2} \times \boldsymbol{E}_{N_{t}}\right) \boldsymbol{e}_{3} d s= \\
& \int_{L_{i}} \boldsymbol{E}_{N_{t}} \boldsymbol{e}_{1} d s=\hat{e}_{i_{N_{t}}}
\end{aligned}
$$

Now the magnetic current density can be calculated from the electric grid voltages by using Eq. (14). The electric currents are located at the same time step like the magnetic currents (see Fig. 4a). It is possible to locate the electric currents at the same time step like the magnetic grid voltages and even to use a time step $\triangle T_{B E M}$ for the electric currents which is larger than the time step given by FIT (see Fig. $4 \mathrm{~b}$ ). The latter decreases calculation time and improves stability because the time step given by the FIT is extremely small.

\subsection{Time step $t=N_{t} \triangle T$}

The calculation of the magnetic grid voltages interconnecting the inhomogeneous and the homogeneous region (see Fig. 3) with the TD-HFIE is equivalent to the use of the so called pulse-test-function:

$$
\int_{S} \boldsymbol{H} d \boldsymbol{s}=\int_{A} \boldsymbol{H} \boldsymbol{\beta}_{\text {pulse }} d a, \text { with } \boldsymbol{\beta}_{\text {pulse }}=\left.\frac{d \boldsymbol{s}}{|d \boldsymbol{s}|}\right|_{\boldsymbol{r} \in S}
$$

The electric and magnetic current density is approximated according to Sect. 2. Thus the magnetic grid voltages can be calculated by combining Eq. (7) and the Fitzgeraldtransformation/duality. The use of a pulse-test-function allows us to calculate the magnetic grid voltages without the need to calculate a term proportional to $1 / r^{2}$. As seen in Fig. 5a the minimum distance between boundary plane and magnetic grid voltages is $0.5 L_{\min }$ which is half of the smallest spatial discretization. In Fig. 5a the distance between the Huygens-surface and the magnetic grid voltages is half a cellsize.

In our numerical example we will use one and a half cellsizes between Huygens-surface and the grid voltages calculated by the TD-HFIE (see Fig. 6a). Due to the Courant condition the minimal temporal retardation can be calculated to:

$\tau_{\text {min }}=\frac{L_{\text {min }}}{c}=\frac{\sqrt{3} c \Delta T}{c}=\sqrt{3} \Delta T>0.5 \triangle T$ 


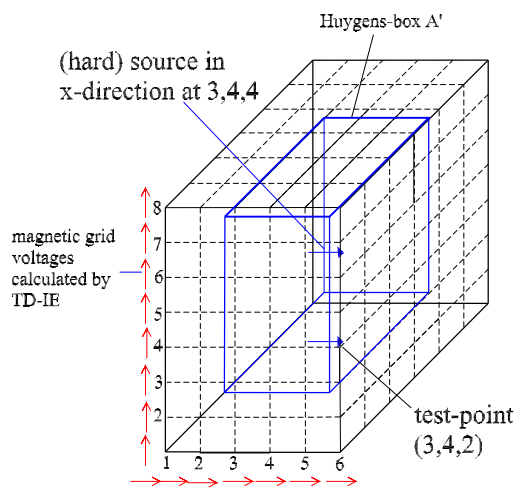

a)

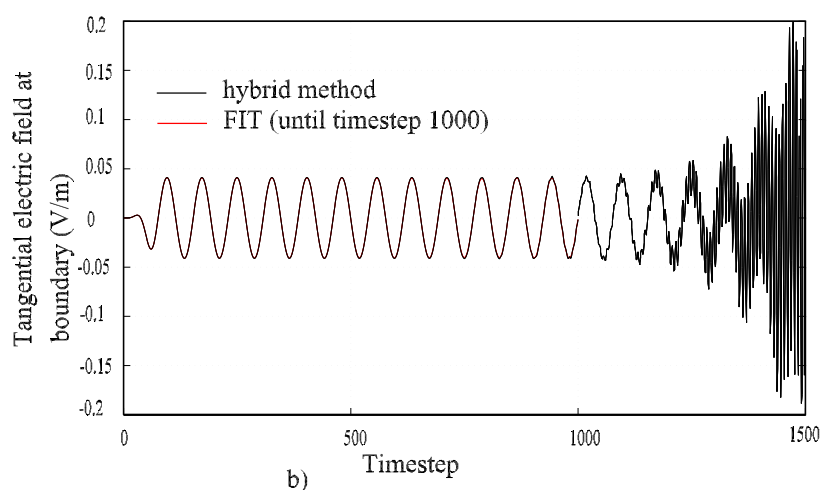

Fig. 6. Geometry and electric fields at the boundary of the FIT-volume.
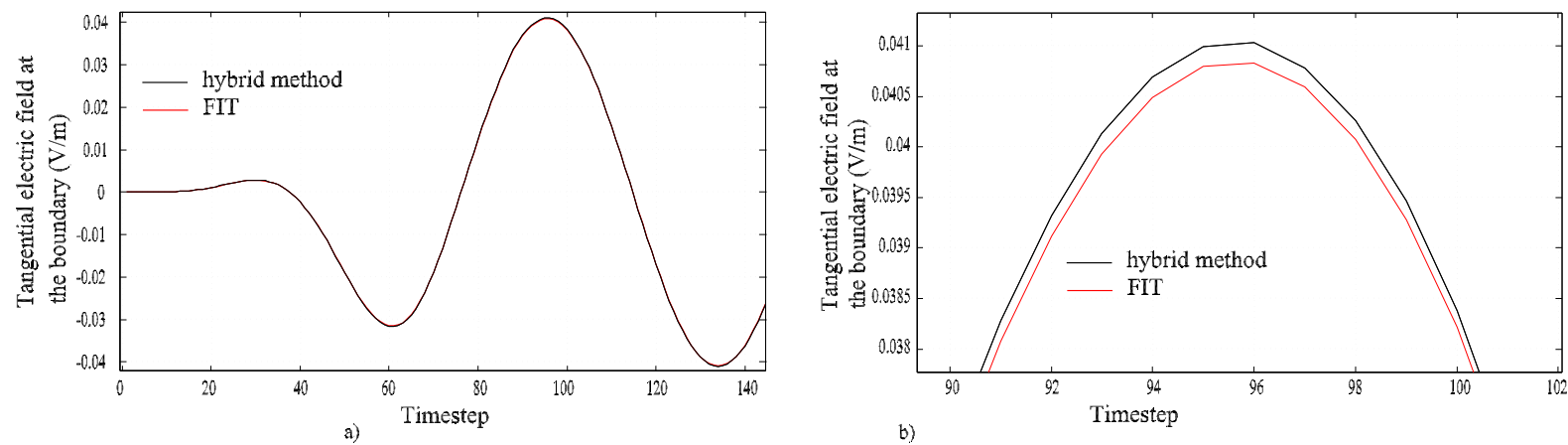

Fig. 7. The electric fields at the boundary of the FIT-volume.

As seen in Fig. 5b the actual magnetic grid voltages are not functions of the actual electric and magnetic currents. Thus the grid voltages can be calculated by using expired currents only.

\subsection{Time step $t=\left(N_{t}+0.5\right) \triangle T$}

If the tangential magnetic grid voltages are known the FITalgorithm can be completed in the interior and on the boundary as usual $\left(t=\left(N_{t}+0.5\right) \triangle T\right)$. Consequently, the electric grid voltages are known in the whole interior and on the boundary. With Eq. (14) the magnetic currents can be calculated in a very efficient way. Anyway, the FIT does not give a relationship between the magnetic and the electric fields in one common plane. At the next time-step $\left(t=\left(N_{t}+1\right) \triangle T\right)$ the magnetic as well as the electric currents at time steps $t=\left(N_{t}+0.5\right) \triangle T$ are needed. The latter are calculated by applying Eq. (10) at $t=\left(N_{t}+0.5\right) \triangle T$. As boundary condition we use the electric fields calculated by FIT (proportional to the magnetic flux as related by Eq. 14), i.e. the fields calculated by the TD-IE must equal the fields calculated by the FIT. Now both electric and magnetic currents are known on the surface $A^{\prime}$.

\section{Numerical results}

As a first numerical evaluation of the hybrid method we simulate an electric source (sinusoidal, $500 \mathrm{MHz}$ ) in the interior of an air filled cube (see Fig. 6a) by impressing one electric grid voltage. This source reflects all incoming waves and so it acts like a small wire. The spatial and temporal discretization is determined by the Courant condition, assuming a $1.1 \mathrm{GHz}$ source and 20 cells per wavelength. The cube is $6 \times 7 \times 8$-cells big. Between the Huygens-surface and the FIT-boundary we put one layer. As an example we calculate one electric grid voltage on the Huygens-surface. The result is compared to a calculation in which a cube with PMCboundary condition is simulated. This simulation contains so many cells in each direction that waves at the boundary can be neglected. The results agree well despite of the extremely small distance between boundary and source. However, after approximately 12 periods the calculation with the hybrid method becomes unstable. Such instabilities are typical for TD-BIMs and the accuracy and stability strongly depends on the numerical evaluation of the matrix $\mathbf{Z}$ and the coefficients $X_{(k, i, j)}$ and $Y_{(k, i, j)}$. So it can expected be that these instabilities can be postponed in time far enough for practical purposes, e.g. by using semi-analytic integration. 


\section{Summary and outlook}

The presented hybrid method combines the "local" FIT and a "global" TD-BIM. The TD-BIM acts like an absorbing boundary condition and allows waves to pass bidirectional. The method can be directly transformed into a frequency domain technique and needs no additional interpolation than the interpolations inherent in FIT. The accuracy and stability can be improved by using semi-analytic integration instead of the implemented numerical integration. The efficiency can be drastically increased by using techniques like the plane wave time domain algorithm (Shanker, 2000).

\section{References}

De Moerlosse, J. and De Zutter, D.: Surface Integral Representation Radiation Boundary Condition for the FDTD-method, IEEE Trans. AP., 41, 890-896, 1993.

Rao, S. M.: Time Domain Electromagnetics, Academic Press, 132, 1999.

Sarkar, T. K., Lee, W., and Rao, S. M.: Analysis of Transient Scattering From Composite Arbitrarily Shaped Complex Structures, IEEE Trans. AP, 48, 1625-1634, 2000.

Shanker, B., Ergin, A., Aygü, K., and Michielssen, E.: Analysis of Transient Electromagnetic Scattering Phenomena Using a TwoLevel Plane Wave Time-Domain Algorithm, IEEE Trans. AP, 48, 510-523, 2000.

Weiland, T.: Time Domain Electromagnetic Field Computation With Finite Difference Methods, International Journal of Numerical Modelling: Electronic Networks, Devices and Fields, 9 , 295-319, 1996. 